\title{
Zeros of the Wigner distribution and the short-time Fourier transform
}

\author{
Karlheinz Gröchenig ${ }^{1}$ (1) - Philippe Jaming ${ }^{2}$ - Eugenia Malinnikova ${ }^{3,4}$ \\ Received: 12 December 2018 / Accepted: 5 November 2019 / Published online: 2 December 2019 \\ (c) The Author(s) 2019
}

\begin{abstract}
We study the question under which conditions the zero set of a (cross-) Wigner distribution $W(f, g)$ or a short-time Fourier transform is empty. This is the case when both $f$ and $g$ are generalized Gaussians, but we will construct less obvious examples consisting of exponential functions and their convolutions. The results require elements from the theory of totally positive functions, Bessel functions, and Hurwitz polynomials. The question of zero-free Wigner distributions is also related to Hudson's theorem for the positivity of the Wigner distribution and to Hardy's uncertainty principle. We then construct a class of step functions $S$ so that the Wigner distribution $W\left(f, \mathbf{1}_{(0,1)}\right)$ always possesses a zero $f \in S \cap L^{p}$ when $p<\infty$, but may be zerofree for $f \in S \cap L^{\infty}$. The examples show that the question of zeros of the Wigner distribution may be quite subtle and relate to several branches of analysis.
\end{abstract}

Keywords Wigner distribution - Short-time Fourier transform · Hudson's theorem · Poly-analytic function · Convexity $\cdot$ Hurwitz polynomial $\cdot$ Totally positive function

Mathematics Subject Classification 42A38 $\cdot 42 \mathrm{~B} 10 \cdot 81 \mathrm{~S} 30$

Karlheinz Gröchenig

karlheinz.groechenig@univie.ac.at

Philippe Jaming

philippe.jaming@gmail.com

Eugenia Malinnikova

eugeniam@stanford.edu

1 Faculty of Mathematics, University of Vienna, Oskar-Morgenstern-Platz 1, 1090 Vienna, Austria

2 Institut de Mathématiques de Bordeaux UMR 5251, Université de Bordeaux, Cours de la Libération, 33405 Talence Cedex, France

3 Department of Mathematical Sciences, Norwegian University of Science and Technology, Alfred Getz vei 1, Trondheim, Norway

4 Department of Mathematics, Stanford University, Building 380, Stanford, CA 94305, USA 


\section{Introduction}

The aim of this paper is to study the zero set of the Wigner distribution of two functions $f, g \in L^{2}(\mathbb{R})$,

$$
W(f, g)(z)=\int_{\mathbb{R}^{d}} f\left(x+\frac{t}{2}\right) \bar{g}\left(x-\frac{t}{2}\right) e^{-2 \pi i\langle\xi, t\rangle} \mathrm{d} t, \quad z=(x, \xi) \in \mathbb{R}^{2 d} .
$$

More precisely, we are investigating whether this zero set can be empty. Results here directly extend to other phase-space representations to which the Wigner transform is closely related. These include the ambiguity function and the short-time Fourier transform $V_{g} f(z)=\langle f, \pi(z) g\rangle_{L^{2}\left(\mathbb{R}^{d}\right)}, \pi(z) g(t)=e^{2 i \pi\langle\xi, t\rangle} g(t-x)$ for $z=(x, \xi) \in$ $\mathbb{R}^{2 d}$ (see Eq. 2).

The zero set of the short-time Fourier transform is important in the study of the generalized Berezin quantization and the injectivity of a general Berezin transform. The thesis of D. Bayer [5], partially published in [6], contains the following result (under a mild condition on $f, g \in L^{2}\left(\mathbb{R}^{d}\right)$ ): If $V_{g} f(z) \neq 0$ for all $z \in \mathbb{R}^{2 d}$, then the mapping $T \rightarrow \mathcal{B} T$, with $z \rightarrow \mathcal{B} T(z)=\langle T \pi(z) g, \pi(z) f\rangle$, is one-to-one on the space of bounded operators on $L^{2}\left(\mathbb{R}^{d}\right)$. The function $(z, w) \mapsto\langle T \pi(z) g, \pi(w) f\rangle$ may be interpreted as a special integral kernel associated to the operator $T$. Bayer's statement asserts that $T$ is uniquely determined by the diagonal of this kernel. The assertation is relevant, because theorems of this type were known only in the context of complex analysis, see, e.g. [10, Cor. 1.70]. Zero-free Wigner distributions occur prominently in $[22,24]$ in a similar context. It is therefore natural to ask for examples that satisfy Bayer's assumptions:

Question Determine (all) pairs $(f, g)$ such that $W(f, g)$ does not vanish.

Clearly, if $f(t)=g(t)=e^{-\pi t^{2}}$ is a Gaussian, then $W(f, g)(x, \xi)=$ $2^{d} e^{-2 \pi\left(x^{2}+\xi^{2}\right)} \neq 0$ everywhere. More generally, if $q, \tilde{q}$ are quadratic polynomials over $\mathbb{C}$ such that $f=e^{-q}, g=e^{-\tilde{q}} \in L^{2}\left(\mathbb{R}^{d}\right)$ (generalized Gaussian), then the Wigner distribution $W(f, g)$ is also zero-free. For a while, we have been unable to produce other pairs $(f, g)$ for which $W(f, g)$ is zero-free which led us to believe that such pairs might not exist. The aim of this paper is to show that our belief was false by providing several examples of Wigner distributions without zeros. In doing so, we explore several connections with other areas of analysis. We restrict our attention mostly to the case $d=1$. Although we cannot offer a coherent theory of zero-free Wigner distributions, we feel that the above question is of interest in itself and warrants a deeper analysis. Part of the appeal of this problem comes from the connection to different branches of analysis. In our quest for zero-free Wigner distributions we touched the following topics in analysis.

(i) Total positivity: Most of our examples of zero-free Wigner distributions (or ambiguity functions) are obtained from the basic example of the one-sided exponential function $e^{-a t} \mathbf{1}_{(0, \infty)}(t)$ by convolution. These happen to be totally positive functions, as follows from the fundamental classification of totally positive functions by Schoenberg [28]. However, not all totally positive functions possess a zero- 
free ambiguity function, as can be seen from the symmetric exponential function $e^{-a|x|}$.

(ii) Bessel functions and Hurwitz polynomials: The Wigner distribution of the functions $f_{n}(t)=t^{n} e^{-t} \mathbf{1}_{(0, \infty)}(t)$ contains certain Bessel functions as a factor. Moreover, to verify that the ambiguity function of $f_{n}$ is zero-free, one needs to know that certain polynomials have all their zeros in the left half-plane, i.e., whether they are Hurwitz polynomials. We answer this question by recourse to the classical properties of Bessel functions.

(iii) Hudson's theorem: The non-vanishing of a Wigner distribution seems inherently related to Hudson's theorem which asserts that a Wigner distribution is nonnegative, if and only if both $f$ and $g$ are generalized Gaussians [15]. Indeed, variations of the proof of Hudson's theorem yield the following statement: If Re $W(f, g)$ does not have any zeros, then $f$ and $g$ must be generalized Gaussians. Similar statement holds for the imaginary part of $W(f, g)$. For all other functions, the real and imaginary parts of the Wigner distribution must change sign, so the zero sets contain some hypersurfaces and the question is when these hypersurfaces do not intersect.

(iv) Poly-analytic functions: We also consider the case when both functions $f$ and $g$ are finite linear combinations of Hermite functions. We are lead to investigate the zeros of certain poly-analytic functions. Results of Balk [4] yield some hints about the zero set in this case. However, this direction does not seem to lead to new examples of zero-free Wigner distributions. We conjecture that no such examples exist in the class of finite linear combinations of Hermite functions.

(v) Convexity and almost periodicity: As a last class of functions we consider pairs $f, g$ where one of the functions, say $g$, is a characteristic function of an interval. We conjecture that for any choice of $f \in L^{2}(\mathbb{R})$ the Wigner distribution $W(f, g)$ has zeros. To support our conjecture we study the following particular case. Let $g=\mathbf{1}_{(0,1)}$ and choose $f$ to be a step function with discontinuities on $\mathbb{Z} \cup(\alpha+\mathbb{Z})$ for irrational $\alpha$. If $f \in L^{p}$ for $p<\infty$, the Wigner distribution $W\left(f, \mathbf{1}_{(0,1)}\right)$ must always have a zero. However, we will construct a delicate example of a step function $f$ in $L^{\infty}$ for which the corresponding Wigner distribution $W\left(f, \mathbf{1}_{(0,1)}\right)$ does not have any zeros. This example shows that the non-existence of zeros of the Wigner distribution may be quite subtle and may depend sensitively on integrability or smoothness properties of the function classes considered. In this part we use convexity and almost periodicity as tools.

The article is organized as follows: in Sect. 2 we collect some of the properties of the Wigner distribution and the ambiguity function. We list examples we found for which the Wigner distribution is zero-free in Sects. 3 and 4. In Sect. 5 we study the connection of zero-free Wigner distributions to Hudson's theorem. Open questions and interesting connections of the problem to other areas of analysis are discussed in Sects. 4-7. In Sect. 6 we consider $f$ and $g$ to be finite linear combinations of Hermite functions and relate the corresponding Wigner distribution to the theory of poly-analytic functions. Section 7 is devoted to the case when $g$ is the characteristic function of an interval. 


\section{Some properties of the Wigner transform}

The Wigner transform is closely related to two other transforms in time-frequency analysis. The first one is the short-time Fourier transform

$$
V_{g} f(x, \xi)=\int_{\mathbb{R}^{d}} f(t) \overline{g(t-x)} e^{-2 \pi i\langle\xi, t\rangle} \mathrm{d} t
$$

A simple computation shows that

$$
W(f, g)(x, \xi)=e^{4 \pi i\langle x, \xi\rangle} V_{I g} f(2 x, 2 \xi),
$$

with $I g(t)=g(-t)$. The second transform is the ambiguity function which is a slighlty more symmetric version of the short-time Fourier transform

$$
A(f, g)=\int_{\mathbb{R}^{d}} f\left(t+\frac{x}{2}\right) \overline{g\left(t-\frac{x}{2}\right)} e^{-2 i \pi\langle\xi, t\rangle} \mathrm{d} t .
$$

These transforms are related by the formulas

$$
A(f, g)=e^{i \pi\langle\xi, x\rangle} V(f, g) \text { and } A(f, g)(x, \xi)=\frac{1}{2} W(f, I g)\left(\frac{x}{2}, \frac{\xi}{2}\right)
$$

Thus a pair $(f, g)$ for which $A(f, g)$ does not vanish also provides a pair $(f, g)$ for which the short-time Fourier transform and a pair $(f, I g)$ for which the Wignertransform does not vanish.

Let us now list the invariance properties of the ambiguity function. To do so, we recall that, for $z=(x, \xi) \in \mathbb{R}^{2 d}$, the phase-space shift of $f \in L^{2}\left(\mathbb{R}^{d}\right)$ along $z$ is defined by $\pi(z) f(t)=e^{2 \pi i\langle\xi, t\rangle} f(t-x)$. To every symplectic matrix $\mathcal{A} \in \operatorname{Sp}(d, \mathbb{R})$ one can then associate a unitary operator $\mu(\mathcal{A})$ acting on $L^{2}\left(\mathbb{R}^{d}\right)$ such that

$$
\pi(\mathcal{A} z)=\mu(\mathcal{A}) \pi(z) \mu(\mathcal{A})^{*} \quad \forall z \in \mathbb{R}^{2 d} .
$$

Here $\mu(\mathcal{A})$ is determined only up to a phase factor and is called a metaplectic operator. The existence of metaplectic operators follows already from the Stone-von Neumann theorem [10, Thm. 1.50] and is all we need here. For the much deeper aspects of these operators and the subtle construction of the metaplectic representation we refer to [10] and the recent book [9]. For Theorem 5.4 we need the following formulas about the interaction between the Wigner distribution and the metaplectic operators.

Lemma 2.1 Let $f, g \in L^{2}\left(\mathbb{R}^{d}\right), w=(a, b), w^{\prime}=\left(a^{\prime}, b^{\prime}\right) \in \mathbb{R}^{2 d}$, and $\mathcal{A} \in \operatorname{Sp}(d, \mathbb{R})$. Then for all $z \in \mathbb{R}^{2 d}$

$$
\begin{gathered}
W\left(\pi(w) f, \pi\left(w^{\prime}\right) g\right)(z)=e^{2 i \pi\left\langle w-w^{\prime}, z\right\rangle+i \pi\left\langle b+b^{\prime}, a-a^{\prime}\right\rangle} W(f, g)\left(z-\frac{w+w^{\prime}}{2}\right) \\
W(\mu(\mathcal{A}) f, \mu(\mathcal{A}) g)(z)=W(f, g)\left(\mathcal{A}^{*} z\right) .
\end{gathered}
$$


See [10], Prop. (1.92), for (5) (with slightly different normalizations), and [10], Prop. (4.28), for (6), or the general references [9,12].

As a consequence, if $W(f, g)$ has no zeros, then the same is true for $W(\pi(w) f$, $\left.\pi\left(w^{\prime}\right) g\right)$ and $W(\mu(\mathcal{A}) f, \mu(\mathcal{A}) g)$. Thus every example of a zero-free Wigner distribution yields a whole class of related examples. Note that when $\mathcal{A}=J=\left(\begin{array}{cc}0 & -I \\ I & 0\end{array}\right)$, $\mu(\mathcal{A}) f$ is the Fourier transform of $f$.

We will use a further property of the ambiguity function. For $\xi \in \mathbb{R}^{d}$, let us write $M_{\xi} f(t)=\pi(0, \xi) f(t)=e^{2 i \pi\langle\xi, t\rangle} f(t)$. Then

$$
A(f, g)(x, \xi)=\left(M_{-\xi / 2} f\right) *\left(M_{\xi / 2} g^{*}\right)(x)
$$

where $g^{*}(t)=\overline{g(-t)}$. As a consequence

$$
\begin{aligned}
A\left(f_{1} * f_{2}, g_{1} * g_{2}\right)(x, \xi) & =\left(A\left(f_{1}, g_{1}\right)(\cdot, \xi) *{ }_{1} A\left(f_{2}, g_{2}\right)(\cdot, \xi)\right)(x) \\
& :=\int_{\mathbb{R}^{d}} A\left(f_{1}, g_{1}\right)(t, \xi) A\left(f_{2}, g_{2}\right)(x-t, \xi) \mathrm{d} t
\end{aligned}
$$

Finally we cite the version of Hardy's uncertainty principle for the Wigner distribution taken from [14].

Lemma 2.2 (i) If $|W(f, f)(z)| \leq C e^{-2 \pi|z|^{2}}=C^{\prime} W\left(h_{0}, h_{0}\right)(z)$ for all $z \in \mathbb{R}^{2 d}$, then $f=c h_{0}$.

(ii) Let $\gamma \in L^{2}\left(\mathbb{R}^{d}\right)$ be a generalized Gaussian and $\psi \in L^{2}\left(\mathbb{R}^{d}\right)$. If $W(\psi, \psi)(z) \leq$ $W(\gamma, \gamma)(z)$ for all $z \in \mathbb{R}^{2 d}$, then $\psi=c \gamma$.

Proof (i) is Hardy's uncertainty principle for the Wigner distribution as proved in [14] (Lemma 3.3 and Remark 3.4).

(ii) follows from (i). We recall that every generalized Gaussian $\gamma=e^{Q(x)} \in L^{2}\left(\mathbb{R}^{d}\right)$ with a quadratic polynomial $Q$ can be written in the form $\gamma=\pi(w) \mu(\mathcal{A}) h_{0}$, where $h_{0}(x)=2^{d / 4} e^{-\pi|x|^{2}}$ is the normalized Gaussian, see e.g., [10], Prop. (4.73).

Let $\psi_{0} \in L^{2}\left(\mathbb{R}^{d}\right)$ such that $\psi=\pi(w) \mu(\mathcal{A}) \psi_{0}$. Using (5) and (6), we obtain

$$
\begin{aligned}
\left|W\left(\psi_{0}, \psi_{0}\right)\left(\mathcal{A}^{*}(z-w)\right)\right| & =|W(\psi, \psi)(z)| \\
& \leq C W(\gamma, \gamma)(z)=C^{\prime} W\left(h_{0}, h_{0}\right)\left(\mathcal{A}^{*}(z-w)\right) \quad z \in \mathbb{R}^{2 d} .
\end{aligned}
$$

By (i) this implies that $\psi_{0}=c h_{0}$ and consequently $\psi=c \gamma$.

\section{Examples}

In this section we give several examples of pairs of functions in dimension 1 for which the ambiguity function does not vanish. 
Example 1 The first example is the Gaussian function. Write $\gamma_{a}(t)=e^{-a \pi t^{2}}$ with $t \in$ $\mathbb{R}$ and $a>0$. Then a direct, well known computation shows that, if $\operatorname{Re}(a), \operatorname{Re}(b)>0$,

$$
A\left(\gamma_{a}, \gamma_{b}\right)=(a+b)^{-1 / 2} \exp \left(-\pi \frac{a b x^{2}+\xi^{2}}{a+b}+i \frac{a-b}{a+b} x \xi\right)
$$

so that $A\left(\gamma_{a}, \gamma_{b}\right)$ does not vanish.

Using tensorisation and invariance properties of the Wigner distribution, it follows that, if $f, g \in L^{2}\left(\mathbb{R}^{d}\right.$ ) are generalized Gaussians $f=e^{-q_{1}}, g=e^{-q_{2}}$ (for some quadratic polynomial), then $A(f, g)$ does not vanish on $\mathbb{R}^{d}$.

A second family of examples is given by the one-sided exponential.

Example 2 For $a>0$, let $\eta_{a}(t)=e^{-a t} \mathbf{1}_{(0,+\infty)}(t)$. Then, for $a, b>0$

$$
\begin{aligned}
A\left(\eta_{a}, \eta_{b}\right)(x, \xi) & =\exp \left(-\frac{a-b}{2} x-\frac{a+b}{2}|x|\right) \frac{e^{-i \pi \xi|x|}}{a+b+2 i \pi \xi} \\
& =\eta_{a, b}(x) \frac{e^{-i \pi \xi|x|}}{a+b+2 i \pi \xi}
\end{aligned}
$$

where $\eta_{a, b}(x)=(b-a)^{-1} \eta_{a} * I \eta_{b}(x)=\left\{\begin{array}{ll}e^{-a x} & \text { when } x \geq 0 \\ e^{b x} & \text { when } x<0\end{array}\right.$. See also [19]. In particular,

$$
A\left(\eta_{a}, \eta_{a}\right)(x, \xi)=\frac{e^{-(a+i \pi \xi)|x|}}{2(a+i \pi \xi)}
$$

does not have any zeros. Since $A(I f, I g)(x, \xi)=A(f, g)(-x,-\xi)$, the ambiguity function of the one-sided exponential $I \eta_{b}, b>0$, is

$$
A\left(I \eta_{b}, I \eta_{b}\right)(x, \xi)=\frac{e^{-(b-i \pi \xi)|x|}}{2(b-i \pi \xi)}
$$

and is also zero-free.

However, the ambiguity function $A\left(\eta_{a}, I \eta_{b}\right)(x, \xi)=0$ for $x \leq 0$, because in this case $t \mapsto \eta_{a}(t+x / 2)$ and $t \mapsto I \eta_{b}(t-x / 2)$ have disjoint supports.

Example 3 Next we compute the ambiguity function of convolutions of exponentials of the form $f=\eta_{a} * \eta_{b}$ or $f=\eta_{a} * I \eta_{b}$ for $a, b>0$. For a compact formula we write $\eta_{-a}=I \eta_{a}$.

Lemma 3.1 Let $a, b>0, a \neq b$. Then

$$
\begin{aligned}
& A\left(\eta_{a} * \eta_{ \pm b}, \eta_{a} * \eta_{ \pm b}\right)(x, \xi) \\
& \quad=\frac{1}{2\left(b^{2}-a^{2}\right)+4 \pi i( \pm b-a) \xi}\left(\frac{e^{-(a+\pi i \xi|x|)}}{a+\pi i \xi}-\frac{e^{-(b \pm \pi i \xi)|x|}}{b \pm \pi i \xi}\right) .
\end{aligned}
$$

Furthermore, $A\left(\eta_{a} * \eta_{ \pm b}, \eta_{a} * \eta_{ \pm b}\right)$ has no zeros. 
Proof To facilitate calculations, we set $\psi_{u}(x)=e^{-u|x|}$ for $u \in \mathbb{C}$. Given $a, b>0$ set $u=a+\pi i \xi, v=b+\pi i \xi$. In this notation, (9) with $a=b$ becomes

$$
A\left(\eta_{a}, \eta_{a}\right)(x, \xi)=\frac{1}{2 u} \psi_{u}(x) \quad \text { and } \quad A\left(\eta_{-b}, \eta_{-b}\right)(x, \xi)=\frac{1}{2 \bar{v}} \psi_{\bar{v}}(x) .
$$

To find the ambiguity function of $f=\eta_{a} * \eta_{b}$, we use (7) and find that

$$
A(f, f)(x, \xi)=\frac{\psi_{u} * \psi_{v}(x)}{4 u v}
$$

Then for $x \geq 0$ we obtain

$$
\begin{aligned}
\psi_{u} * \psi_{v}(x) & =\int_{-\infty}^{0} e^{u t-v(x-t)} \mathrm{d} t+\int_{0}^{x} e^{-u t-v(x-t)} \mathrm{d} t+\int_{x}^{+\infty} e^{-u t+v(x-t)} \mathrm{d} t \\
& =\frac{e^{-v x}}{u+v}+\frac{e^{-u x}-e^{-v x}}{v-u}+\frac{e^{-u x}}{u+v} \\
& =e^{-u x}\left(\frac{1}{v+u}+\frac{1}{v-u}\right)+e^{-v x}\left(\frac{1}{v+u}-\frac{1}{v-u}\right) \\
& =\frac{2}{v^{2}-u^{2}}\left(v e^{-u x}-u e^{-v x}\right) .
\end{aligned}
$$

Since $\psi_{u}$ is even, so is $\psi_{u} * \psi_{v}$, and then (12) yields

$$
A(f, f)(x, \xi)=\frac{1}{2\left(v^{2}-u^{2}\right)}\left(\frac{e^{-u|x|}}{u}-\frac{e^{-v|x|}}{v}\right),
$$

from which (11) follows by substituting the values for $u$ and $v$.

For the case $f=\eta_{a} * \eta_{-b}$, according to (10) we only have to replace $v$ by $\bar{v}$ in the above derivation, whence (11) follows for $f$.

Now assume that $A\left(\eta_{a} * \eta_{b}, \eta_{a} * \eta_{b}\right)(x, \xi)=0$. Since $a \neq b$, the denominator $v^{2}-u^{2} \neq 0$ and (13) implies that

$$
\frac{e^{-u|x|}}{u}=\frac{e^{-v|x|}}{v},
$$

or

$$
e^{(b-a)|x|}=e^{(v-u)|x|}=\frac{u}{v}=\frac{a+\pi i \xi}{b+\pi i \xi} .
$$

For $b>a$, the left-hand side is greater than 1, whereas the modulus of the right-hand side is less than 1 , which is impossible. Thus $A\left(\eta_{a} * \eta_{b}, \eta_{a} * \eta_{b}\right)$ does not have any zeros. Likewise for the case $b<a$.

Similarly, if $A\left(\eta_{a} * \eta_{-b}, \eta_{a} * \eta_{-b}\right)(x, \xi)=0$, then

$$
\frac{e^{-u|x|}}{u}=\frac{e^{-\bar{v}|x|}}{\bar{v}},
$$


or

$$
e^{(b-a-2 \pi i \xi)|x|}=e^{(\bar{v}-u)|x|}=\frac{u}{\bar{v}}=\frac{a+\pi i \xi}{b-\pi i \xi} .
$$

Again for $b>a$, the modulus of the left-hand side is greater than 1, whereas the modulus of the right-hand side is less than 1 , which is not possible. Thus $A\left(\eta_{a} *\right.$ $\left.\eta_{-b}, \eta_{a} * \eta_{-b}\right)$ does not have any zeros.

The case $a=b$ and $\eta_{a} * \eta_{a}=t e^{-a t} \mathbf{1}_{(0, \infty)}$ is treated below. The case of $\eta_{a} * \eta_{-a}=$ $e^{-a|x|}$ is particularly interesting, as its Fourier transform is the Poisson kernel. In this case (11) holds for $\xi \neq 0$ and requires some easy modification for $\xi=0$. If $\xi \neq 0$, then (16) turns into

$$
e^{-2 \pi i \xi|x|}=e^{(\bar{v}-u)|x|}=\frac{u}{\bar{v}}=\frac{a+\pi i \xi}{a-\pi i \xi}
$$

This equation is solvable, and therefore the ambiguity function of $e^{-a|x|}$ must have zeros. In Corollary 5.2 we will give a different explanation for this fact.

It is also possible to derive a formula for the ambiguity function of higher convolutions of the form $\eta_{a_{1}} * \eta_{a_{2}} * \cdots * \eta_{a_{n}}$ for distinct values of $a_{j}>0$. These expressions are much more complicated, and the analysis of their zeros requires a separate investigation.

Example 4 Let $a>0$ and $f(t)=\eta_{a} * \eta_{a}(t)=t \eta_{a}(t)$. A direct computation shows that

$$
A\left(f, \eta_{a}\right)(x, \xi)=\frac{x \mathbf{1}_{(0,+\infty)}(x) e^{-a(1+i \pi \xi) x}}{2(a+i \pi \xi)}+\frac{e^{-a(1+i \pi \xi)|x|}}{4(a+i \pi \xi)^{2}}
$$

Obviously, $A\left(f, \eta_{a}\right)(x, \xi)$ can not vanish if $x \leq 0$, while if $x \geq 0$, it is enough to notice that $2 x(a+i \pi \xi)+1$ can not vanish.

Example 5 Let $\gamma_{a, b}(t)=\exp \left(a t-b e^{t}\right)$, where $a, b>0$, then $\gamma_{a, b} \in L^{2}(\mathbb{R})$ and we claim that $A\left(\gamma_{a, b}, \gamma_{a, b}\right)$ does not vanish. A straightforward computation (with the substitution $s=2 b \cosh \frac{x}{2} e^{t}$ ) shows that

$$
\begin{aligned}
A\left(\gamma_{a, b}, \gamma_{a, b}\right)(x, \xi) & =\left(2 b \cosh \frac{x}{2}\right)^{-2 a+2 \pi i \xi} \int_{0}^{\infty} s^{2 a-1-2 \pi i \xi} e^{-s} d s \\
& =\left(2 b \cosh \frac{x}{2}\right)^{-2 a+2 \pi i \xi} \Gamma(2 a-2 \pi i \xi) .
\end{aligned}
$$

The gamma function is well defined in the right half-plane and does not have zeros, so $A\left(\gamma_{a, b}, \gamma_{a, b}\right)$ never vanishes. A similar computation shows that

$$
A\left(\gamma_{a, b}, \gamma_{c, d}\right)=e^{(a-c) x / 2}\left(b e^{x / 2}+d e^{-x / 2}\right)^{-(a+c)+2 \pi i \xi} \Gamma(a+c-2 \pi i \xi),
$$

and it has no zeros either. 
One can now use the invariance properties of the ambiguity function to obtain more examples. For instance, using the Fourier transform, one obtains the following examples.

Example 6 Let us now write, for $a \neq 0, c_{a}(t)=\frac{1}{a+2 i \pi t}$ and note that $c_{a}$ is the Fourier transform of $\eta_{a}$ when $a>0$. It follows that $A\left(c_{a}, c_{b}\right)$ does not vanish for $a, b>0$. Furthermore, except for the Poisson kernel $\frac{1}{a^{2}+4 \pi^{2} t^{2}}$ all rational functions of the form $c_{a}(t) c_{b}( \pm t)=\frac{1}{a+2 i \pi t} \frac{1}{b \pm 2 i \pi t}$ possess a non-vanishing ambiguity function, because they are the Fourier transform of $\eta_{a} * \eta_{b}$ or of $\eta_{a} * I \eta_{b}$.

Outlook and context All functions in Examples 1-5 are totally positive functions or related to such a function. A function $g: \mathbb{R} \rightarrow \mathbb{R}$ is called totally positive (or a Polya frequency function), if for every choice of real numbers $x_{1}<x_{2}<\cdots<x_{n}$ and $y_{1}<y_{2}<\cdots<y_{n}$ with $n \in \mathbb{N}$ the inequality

$$
\operatorname{det}\left(g\left(x_{j}-y_{k}\right)\right)_{j, k=1, \ldots, n} \geq 0
$$

holds. According to a fundamental characterization of Schoenberg [28] the Fourier transform of a totally positive function in $L^{1}(\mathbb{R})$ can be factored as follows:

$$
\hat{g}(\xi)=e^{-\gamma \pi \xi^{2}} e^{2 \pi i \delta \xi} \prod_{j=1}^{\infty}\left(1+2 \pi i \delta_{j} \xi\right)^{-1} e^{2 \pi i \delta_{j} \xi},
$$

with $\delta, \delta_{j} \in \mathbb{R}, 0 \leq \gamma$, and $\sum_{j=1}^{\infty} \delta_{j}^{2}<\infty$. Clearly, every Gaussian $\gamma_{a}(t)=$ $e^{-a \pi t^{2}}, a>0$, is totally positive, and the complex Gaussian $e^{-(a+i b) \pi t^{2}}$ is obtained from $\gamma_{a}$ by a metaplectic operator. In Examples 2 and 3 we have analyzed the ambiguity function of the simplest totally positive functions, corresponding to the factorization $\hat{g}(\xi)=\prod_{j=1}^{L}\left(1+2 \pi i \delta_{j} \xi\right)^{-1}$ for $L=1$ and $L=2$. These are just the functions $\eta_{a}, \eta_{a} * \eta_{b}, \eta_{a} * I \eta_{b}$. By Schoenberg's analysis [28, Sec. 18] the function $e^{a x-e^{a x}}$ for $a \neq 0$ occurring in Example 5 is also totally positive.

At this time the role of totally positive functions in the classification of zero-free ambiguity functions remains unclear. Among all explicit examples considered, only the symmetric exponential $e^{-a|x|}$ has an ambiguity function with zeros. More generally, the ambiguity function of every even totally positive function has a zero. See also Corollary 5.2 below.

\section{Totally positive functions and Hurwitz polynomials}

In this section we study a different class of totally positive functions and its relation to Bessel functions and Hurwitz polynomials. Let

$$
f_{n}(t)=t^{n} e^{-t} \mathbf{1}_{(0, \infty)}(t)=t^{n} \eta_{1}(t) .
$$


This is a totally positive function with Fourier transform $\hat{f}(\xi)=(1+2 \pi i \xi)^{-n}$ [28] and can also be written as $n ! \eta_{1} * \cdots * \eta_{1}$ ( $n$ times). Its ambiguity function can be explicitly calculated as follows. Let $A_{n}$ be the polynomial of degree $n$ defined by

$$
A_{n}(z)=\sum_{k=0}^{n}\left(\begin{array}{l}
n \\
k
\end{array}\right)(n+k) ! z^{n-k}
$$

Lemma 4.1 The ambiguity function of $f_{n}(t)=t^{n} e^{-t} \mathbf{1}_{(0, \infty)}(t)$ is equal to

$$
A\left(f_{n}, f_{n}\right)(x, \xi)=e^{-|x|(1+i \pi \xi)} \frac{1}{(2+2 \pi i \xi)^{2 n+1}} A_{n}(|x|(2+2 \pi i \xi))
$$

Proof We rewrite the ambiguity function as follows:

$$
\begin{aligned}
A\left(f_{n}, f_{n}\right)(x, \xi)= & \int_{\mathbb{R}}\left(t+\frac{x}{2}\right)^{n} e^{-(t+x / 2)} \mathbf{1}_{\mathbb{R}^{+}}\left(t+\frac{x}{2}\right) \\
& \times\left(t-\frac{x}{2}\right)^{n} e^{-(t-x / 2)} \mathbf{1}_{\mathbb{R}^{+}}\left(t-\frac{x}{2}\right) e^{-2 \pi i \xi t} \mathrm{~d} t \\
= & \int_{|x| / 2}^{\infty}\left(t+\frac{x}{2}\right)^{n}\left(t-\frac{x}{2}\right)^{n} e^{-(2+2 \pi i \xi) t} \mathrm{~d} t,
\end{aligned}
$$

since we need both $t>x / 2$ and $t>-x / 2$. For $x \neq 0$, we use the change of variables

$$
u=\frac{t}{|x|}-\frac{1}{2}
$$

(so that $t=|x| u+|x| / 2$ ) to rewrite the integral as

$$
\begin{aligned}
A\left(f_{n}, f_{n}\right)(x, \xi) & =|x|^{2 n+1} \int_{0}^{\infty} u^{n}(u+1)^{n} e^{-(|x| u+|x| / 2)(2+2 \pi i \xi)} \mathrm{d} u \\
& =e^{-|x|(1+\pi i \xi)}|x|^{2 n+1} \int_{0}^{\infty} u^{n}(u+1)^{n} e^{-u|x|(2+2 \pi i \xi)} \mathrm{d} u
\end{aligned}
$$

The integral is just the Laplace transform of the polynomial

$$
u^{n}(u+1)^{n}=\sum_{k=0}^{n}\left(\begin{array}{l}
n \\
k
\end{array}\right) u^{n+k}
$$

at $\zeta=|x|(2+2 \pi i \xi)$. Since $\mathcal{L}\left\{u^{k}\right\}(z)=\int_{0}^{\infty} u^{k} e^{-u z} d u=k ! z^{-k-1}$, we obtain

$$
\int_{0}^{\infty} u^{n}(u+1)^{n} e^{-u z} d u=\sum_{k=0}^{n}\left(\begin{array}{l}
n \\
k
\end{array}\right)(n+k) ! z^{-n-k-1}=z^{-2 n-1} A_{n}(z)
$$


For $x=0$, the ambiguity function is

$$
\begin{aligned}
A\left(f_{n}, f_{n}\right)(0, \xi) & =\int_{0}^{\infty} t^{2 n} e^{-t(2+2 \pi i \xi)} \mathrm{d} t \\
& =\mathcal{L}\left\{u^{2 n}\right\}(2+2 \pi i \xi)=(2 n) !(2+2 \pi i \xi)^{-2 n-1}
\end{aligned}
$$

With $\zeta=|x|(2+2 \pi i \xi)$ and $|x|^{2 n+1} \zeta^{-2 n-1}=(2+2 \pi i \xi)^{-2 n-1}$, the final formula for the ambiguity function on $\mathbb{R}^{2}$ is

$$
A\left(f_{n}, f_{n}\right)(x, \xi)=e^{-|x|(1+i \pi \xi)} \frac{1}{(2+2 \pi i \xi)^{2 n+1}} A_{n}(|x|(2+2 \pi i \xi))
$$

To show that the ambiguity function of $f$ does not vanish, we need to know that $A_{n}$ has no zeros on the right half-plane. A polynomial whose roots all have negative real part, is called a Hurwitz polynomial or a stable polynomial. For small $n$ one can check the stability of $A_{n}$ directly. For larger $n$, one could check the stability of $A_{n}$ in principle with the Routh-Hurwitz criterion [11, pp. 225-230] and [29]. For this purpose, we associate to a polynomial $p(t)=a_{0} t^{n}+a_{1} t^{n-1}+\cdots+a_{n}, a_{0}>0$ the $n \times n$ Hurwitz matrix

$$
\mathcal{H}=\left(a_{2 j-i}\right)_{1 \leq i, j \leq n}=\left(\begin{array}{cccccc}
a_{1} & a_{3} & a_{5} & a_{7} & \cdots & 0 \\
a_{0} & a_{2} & a_{4} & a_{6} & \cdots & 0 \\
0 & a_{1} & a_{3} & a_{5} & \cdots & 0 \\
0 & a_{0} & a_{2} & a_{4} & \cdots & 0 \\
\vdots & \vdots & \vdots & \vdots & & \vdots \\
0 & 0 & 0 & 0 & \cdots & a_{n}
\end{array}\right)
$$

(with the convention $a_{j}=0$ if $j<0$ or $j>n$ ). Then $p$ is a Hurwitz polynomial if and only if all principal minors of $\mathcal{H}$ are positive [11, pp. 225-230]. This criterion implies that the condition $a_{j} a_{j+1}-a_{j-1} a_{j+2}>0$ is necessary for the stability of $p$. On the other hand, there exists an optimal value $\gamma>0, \gamma \approx 2.1479$, such that $a_{j} a_{j+1}>\gamma a_{j-1} a_{j+2}$ is sufficient for $p$ to be stable [20]. One can check that the polynomials $A_{n}$ satisfy the necessary condition, but fail to satisfy the sufficient condition. The decisive hint comes from experimenting with Mathematica. It turns out that

$$
A_{n}(z)=\pi^{-1 / 2} n ! e^{z / 2} z^{n+1 / 2} K_{n+1 / 2}\left(\frac{z}{2}\right)
$$

where $K_{v}, v \in \mathbb{R}$ is the Macdonald function or the modified Bessel function of the second kind $[25,26,31]$. For $v=n+1 / 2$ it is the Laurent polynomial

$$
K_{n+1 / 2}(z)=\left(\frac{\pi}{2 z}\right)^{1 / 2} e^{-z} \sum_{k=0}^{n} \frac{(n+k) !}{k !(n-k) !}(2 z)^{-k},
$$


whence (20) follows immediately. One can also perform the change of variables $s=$ $2 t /|x|$ in the first computation of $A\left(f_{n}, f_{n}\right)$ and directly obtain that

$$
\begin{aligned}
A\left(f_{n}, f_{n}\right)(x, \xi) & =\int_{|x| / 2}^{\infty}\left(t+\frac{x}{2}\right)^{n}\left(t-\frac{x}{2}\right)^{n} e^{-(2+2 \pi i \xi) t} \mathrm{~d} t \\
& =\left(\frac{|x|}{2}\right)^{2 n+1} \int_{1}^{\infty}\left(s^{2}-1\right)^{n} e^{-(1+\pi i \xi)|x| s} \mathrm{~d} s \\
& =\frac{n !}{\sqrt{\pi}}\left(\frac{|x|}{(1+\pi i \xi)}\right)^{n+1 / 2} K_{n+1 / 2}(|x|(1+\pi i \xi))
\end{aligned}
$$

Furthermore, for arbitrary $v \geq 0, K_{v}$ has roots only when $\operatorname{Re} z<0[31,15.7]$. In view of (20) $A_{n}$ is therefore a Hurwitz polynomial [31].

By combining the accumulated knowledge about Bessel functions, we have therefore proved the following result.

Theorem 4.2 The polynomial $A_{n}$ is a Hurwitzpolynomial. Thus the ambiguity function of $f_{n}(t)=t^{n} e^{-t} \mathbf{1}_{(0, \infty)}(t)$ does not have any zeros.

Using the invariance of the problem under metaplectic operators, we see that the function $c_{a}^{n}(t)=\frac{1}{(a+2 i \pi t)^{n}}$ has a zero-free ambiguity function. This follows because $c_{a}^{n}$ is the Fourier transform of $x^{n} e^{-a x} \mathbf{1}_{(0, \infty)}(x)=\eta_{a} * \cdots * \eta_{a}$.

Remark 1 Martin Ehler proposed a different proof that $A_{n}$ is a Hurwitz polynomial. He observed that $A_{n}$ can be interpreted as the hypergeometric function ${ }_{1} F_{1}(n, 2 n ; z)$, whose zeros are known to lie in the left halfplane.

\section{Connection to Hudson's theorem}

We next discuss the connection of non-vanishing Wigner distributions to Hudson's theorem $[15,18]$. This theorem characterizes all non-negative Wigner distributions, even in higher dimensions.

Theorem 5.1 (Hudson-Lieb) Let $f, g \in L^{2}\left(\mathbb{R}^{d}\right)$. Then $W(f, g) \geq 0$, if and only if $f=c g$ for $c>0$ and $g$ is a generalized Gaussian. In this case $W(f, g)(x, \xi)>0$ for all $x, \xi \in \mathbb{R}$.

As the connection to Hudson's theorem is not accidental, it is instructive to review its proof. For full details, we refer to $[12,15,17]$, the cited bilinear version is due to Lieb [23].

One first uses the convolution relation $W(f, f) * W(g, g)=\left|V_{g} f\right|^{2}$ for the Wigner distribution. Using the Gaussian window $h_{0}(t)=2^{d / 4} e^{-\pi|t|^{2}}$ with Wigner distribution $W\left(h_{0}, h_{0}\right)(z)=2^{d} e^{-2 \pi|z|^{2}}$, we may translate the statement about the zeros of the Wigner distribution into a statement about entire functions in the Bargmann-Fock space. The Bargmann transform of $f$ is defined to be

$$
B f(z)=2^{d / 4} e^{-\pi\langle z, z\rangle / 2} \int_{\mathbb{R}^{d}} f(t) e^{-\pi\langle t, t\rangle} e^{2 \pi\langle t, z\rangle} \mathrm{d} t \quad z \in \mathbb{C}^{d},
$$


and it is connected to the short-time Fourier transform with respect to the Gaussian $h_{0}$ via

$$
V_{h_{0}} f(\bar{z})=e^{\pi i\langle x, \xi\rangle} B f(z) e^{-\pi|z|^{2} / 2} .
$$

Here we identify the point $(x, \xi) \in \mathbb{R}^{2 d}$ with $z=x+i \xi \in \mathbb{C}^{d}$. If $W(f, f) \geq 0$, then $\left|V_{h_{0}} f(z)\right|^{2}=W(f, f) * W\left(h_{0}, h_{0}\right)(z)>0$ for all $z \in \mathbb{R}^{2 d}$ and therefore

$$
\left|V_{h_{0}} f(\bar{z})\right|^{2}=|B f(z)|^{2} e^{-\pi|z|^{2}} \neq 0 \quad \text { for all } z \in \mathbb{C}^{d} .
$$

If $V_{h_{0}} f(z) \neq 0$ for all $z \in \mathbb{R}^{2 d}$, then the entire function $B f$ does not have zeros in $\mathbb{C}^{d}$ and thus is of the form $B f(z)=e^{h(z)}$ for some entire function $h$. However, since $V_{h_{0}} f$ is bounded, it follows that $|B f(z)|=e^{\operatorname{Re} h(z)} \leq C e^{\pi|z|^{2} / 2}$. Thus $\operatorname{Re} h(z) \leq C^{\prime}+$ $\pi|z|^{2} / 2$. By Carathéodory's inequality $h$ is then a quadratic polynomial. Inversion of the Bargmann transform yields the conclusion that $f$ must be a generalized Gaussian.

The connection between zero-free Wigner distributions and Hudson's theorem now becomes apparent.

Corollary 5.2 If $f \in L^{2}\left(\mathbb{R}^{d}\right)$ and $W(f, f)(x, \xi) \neq 0$ for all $x, \xi \in \mathbb{R}$, then $f$ is a non-zero multiple of a generalized Gaussian. Likewise, if $V_{I f} f(x, \xi) \neq 0$ for all $x, \xi \in \mathbb{R}$, then $f$ is a generalized Gaussian.

Proof Assume that $W(f, f) \neq 0$ everywhere. Since $W(f, f)$ is real-valued, this means that either $W(f, f)(z)>0$ for all $z \in \mathbb{R}^{2}$ or $W(f, f)(z)<0$ for all $z$, in which case $W(-f, f)>0$ everywhere. By Theorem $5.1 f$ must be a generalized Gaussian.

Next we fix one of the functions and assume that $g(t)=h_{0}(t)=2^{d / 4} e^{-\pi t^{2}}$ is the normalized Gaussian in $\mathbb{R}^{d}$.

Proposition 5.3 If $V_{h_{0}} f$ does not have any zero, then $f$ is a generalized Gaussian.

Proof Since $\left|V_{h_{0}} f(\bar{z})\right|=|B f(z)| e^{-\pi|z|^{2} / 2}$ by (21), the entire function $B f$ does not have zeros in $\mathbb{C}^{d}$. The last part of the proof of Hudson's theorem sketched above now implies that $f$ is a generalized Gaussian.

The standard polarization identity for the Wigner distribution now yields the following result on zeros of the real and imaginary parts of the Wigner distribution.

Theorem 5.4 Let $f, g \in L^{2}\left(\mathbb{R}^{d}\right)$ and assume that the real part of $W(f, g)$ is never zero, $\operatorname{Re} W(f, g)(z) \neq 0$ for all $z \in \mathbb{R}^{2 d}$. Then $f=a \gamma$ and $g=b \gamma$ for a generalized Gaussian $\gamma$ and $a, b \in \mathbb{C}$. Similarly, if $\operatorname{Im} W(f, g)(z) \neq 0$ for all $z \in \mathbb{R}^{2}$, then both $f$ and $g$ are generalized Gaussians.

Proof The polarization identity states that

$$
W(f+g, f+g)-W(f-g, f-g)=4 \operatorname{Re} W(f, g) .
$$


Thus, if $\operatorname{Re} W(f, g)$ does not have any zeros, then we must have either

$$
\begin{aligned}
W(f+g, f+g)(z) & >W(f-g, f-g)(z) \\
\text { or } \quad W(f+g, f+g)(z) & <W(f-g, f-g)(z) \quad \forall z \in \mathbb{R}^{2 d} .
\end{aligned}
$$

We only treat (23), the second case is obtained by replacing $g$ by $-g$.

Proceeding as in the proof of Hudson's theorem, we convolve $W(f \pm g, f \pm g)$ with $W\left(h_{0}, h_{0}\right)(z)=2^{d} e^{-2 \pi|z|^{2}}$. Since convolution with a positive function preserves positivity, (23) implies the strict pointwise inequality

$$
\begin{aligned}
\left|V_{h_{0}}(f+g)\right|^{2} & =W(f+g, f+g) * W\left(h_{0}, h_{0}\right) \\
& >W(f-g, f-g) * W\left(h_{0}, h_{0}\right)=\left|V_{h_{0}}(f-g)\right|^{2} \geq 0 .
\end{aligned}
$$

By Proposition $5.3 f+g$ must be a generalized Gaussian $f+g=\gamma$.

Now, since $W(f-g, f-g) \leq W(\gamma, \gamma)$, Lemma 2.2 (ii) implies that $f-g=c \gamma$ for some $c \in \mathbb{C}$. Solving for $f$ and $g$ we obtain that $f=\frac{1+c}{2} \gamma$ and $g=\frac{1-c}{2} \gamma$, and therefore both $f$ and $g$ are multiples of the same generalized Gaussian.

\section{Polyanalytic functions}

To find examples of zero-free Wigner distributions, one might fix a window function $g$ different from a Gaussian and see if every short-time Fourier transform $V_{g} f$ possesses at least one zero. We pursue this idea in dimension $d=1$ for windows that are finite linear combinations of Hermite functions. This line of thought connects with the theory of poly-analytic functions and raises some new questions.

Let $g=p h_{0}$ where $p$ is a polynomial and $h_{0}(t)=2^{1 / 4} e^{-\pi t^{2}}$ is the normalized Gaussian on $\mathbb{R}$. We can then write $g$ as a finite linear combination of the Hermite functions

$$
g=\sum_{j=0}^{N} c_{j} h_{j}
$$

where $h_{j}(t)=v_{j} e^{\pi t^{2}} \frac{d^{j}}{d t^{j}}\left(e^{-2 \pi t^{2}}\right)$ is the $j$-th Hermite function with normalization $v_{j}$, such that $\left\|h_{j}\right\|_{2}=1$. It is well known that the short-time Fourier transform with respect to such a window is poly-analytic. To prepare the corresponding formulas, fix $n$ and first consider $V_{h_{n}} f$. This short-time Fourier transform can be expressed with the help of the Bargmann transform of $f$ as follows, where $z=x+i \xi \in \mathbb{C}^{d}$ :

$$
V_{h_{n}} f(x,-\xi)=\frac{1}{\sqrt{\pi^{n} n !}} e^{\pi i x \xi} e^{-\pi|z|^{2} / 2} \sum_{k=0}^{n}\left(\begin{array}{l}
n \\
k
\end{array}\right)(-\pi \bar{z})^{n-k} B f^{(k)}(z) .
$$

See $[8,13]$ for some early references of this formula. Now assume that the window is of the form $g=\sum_{n=0}^{N} \sqrt{\pi^{n} n !} c_{n} h_{n}$ and denote the associated polynomial by $P(z)=$ $\sum_{n=0}^{N} c_{n} z^{n}$. Then 


$$
\begin{aligned}
V_{g} f(\bar{z}) & =e^{\pi i x \xi} e^{-\pi|z|^{2} / 2} \sum_{n=0}^{N} \overline{c_{n}} \sum_{k=0}^{n}\left(\begin{array}{l}
n \\
k
\end{array}\right)(-\pi \bar{z})^{n-k} B f^{(k)}(z) \\
& =e^{\pi i x \xi} e^{-\pi|z|^{2} / 2} \sum_{k=0}^{N} \frac{1}{k !}\left(\sum_{n=k}^{N} \overline{c_{n}} \frac{n !}{(n-k) !}(-\pi \bar{z})^{n-k}\right) B f^{(k)}(z) \\
& =e^{\pi i x \xi} e^{-\pi|z|^{2} / 2} \sum_{k=0}^{N} \frac{1}{k !} B f^{(k)}(z) \overline{P^{(k)}(-\pi z)}
\end{aligned}
$$

From this explicit formula we see that the function $F(z)=e^{-\pi i x \xi} e^{\pi|z|^{2} / 2} V_{g} f(\bar{z})$ satisfies the diffential equation

$$
\frac{\partial^{N+1}}{\partial \bar{z}^{N+1}} F=0
$$

In the established terminology, $F$ is poly-analytic of order $N+1$ and $F$ is a version of the poly-analytic Bargmann transform of $f[1,4,30]$. Precisely given $g=\sum_{n=0}^{N} \sqrt{\pi^{n} n !} c_{n} h_{n}$ with associated polynomial $P(z)=\sum c_{n} z^{n}$, we define the poly-analytic Bargmann transform of $f$ with respect to $g$ to be

$$
B_{g} f(z)=\sum_{k=0}^{N} \frac{1}{k !} B f^{(k)}(z) \overline{P^{(k)}(-\pi z)} .
$$

Since $\frac{d^{2}}{d z d \bar{z}}=\frac{1}{4} \Delta$ is the standard Laplacian, (28) can also be written as

$$
B_{g} f(z)=\sum_{k=0}^{N} \frac{1}{k !} 4^{-k} \Delta^{k}(B f(z) \overline{P(-\pi z)}) .
$$

The zero sets of poly-analytic functions are not well understood; in general they are not discrete and are algebraic curves in $\mathbb{C} \simeq \mathbb{R}^{2}$. The zero count is difficult even for such simple equations as $\bar{z}=P(z)$ for a given polynomial $P[4,21]$.

We will use two known, non-trivial results about zeros of poly-analytic functions.

Theorem 6.1 (Balk) If $F$ is poly-analytic and entire, such that its zero set is contained in a bounded set, then $F(z)=e^{h(z)} Q(z, \bar{z})$ for some entire function $h$ and some poly-analytic polynomial $Q(z, \bar{z})$.

See [2] and [27] for a proof. From this result we obtain the following reduction.

Proposition 6.2 Fix $g=p h_{0}$ for some polynomial $p$. If $V_{g} f$ does not have any zeros in $\mathbb{C}$, then $f(x)=q(x) e^{-\left(a^{\prime} x^{2}+b^{\prime} x+c^{\prime}\right)}$ for some polynomial $q$ and $a^{\prime}, b^{\prime}, c^{\prime} \in$ $\mathbb{C}, \operatorname{Re} a^{\prime}>0$.

Proof If the zero set of $V_{g} f$ is empty, then the zero set of the poly-analytic Bargmann transform $B_{g} f$ is also empty (and thus bounded). By Balk's theorem $B_{g} f$ factors 
into an entire function without zeros and a poly-analytic polynomial $Q(z, \bar{z})$, i.e., $B_{g} f(z)=e^{h(z)} Q(z, \bar{z})$ for $h$ entire. Consequently, by (26) we see that

$$
B_{g} f(z)=\sum_{k=0}^{n} \frac{1}{k !} B f^{(k)}(z) \overline{P^{(k)}(-\pi z)}=e^{h(z)} Q(z, \bar{z}) .
$$

Assume that $P(z)=z^{n}+\mathcal{O}\left(z^{n-1}\right)$ and $Q(z, \bar{z})=\sum_{j=0}^{m} p_{j}(z) \bar{z}^{j}$. Comparing the highest term in $\bar{z}$ in (29), we conclude that $n=m$ and that

$$
(-\pi)^{n} B f(z)=e^{h(z)} p_{n}(z) .
$$

Since $B f$ is in the Bargmann-Fock space, it grows at most like $e^{\pi|z|^{2} / 2}$. We conclude that $\operatorname{Re} h(z) \leq c+\pi|z|^{2} / 2$ and therefore $h(z)$ must be a quadratic polynomial $h(z)=$ $-\left(a z^{2}+b z+c\right)$ by Caratheodory's theorem. We thus have $B f(z)=e^{-\left(a z^{2}+b z+c\right)} p_{n}(z)$ and

$$
\begin{aligned}
B f(i y) e^{-\pi y^{2} / 2} & =V_{h_{0}} f(0,-y)=\int_{\mathbb{R}} f(t) e^{-\pi t^{2}} e^{2 \pi i t y} \mathrm{~d} t \\
& =\widehat{\left(f h_{0}\right)}(-y)=e^{-a y^{2}-i b y-c} p_{n}(i y) .
\end{aligned}
$$

Now observe that the inverse Fourier transform of the generalized Gaussian $e^{-a y^{2}-i b y-c}$ is again a generalized Gaussian $e^{r(x)}$, and the polynomial $p_{n}(i y)$ turns into the differential operator $p_{n}\left(\frac{1}{2 \pi} \frac{d}{d x}\right)$. Consequently, $f h_{0}=p_{n}\left(\frac{1}{2 \pi} \frac{d}{d x}\right) e^{r(x)}$ and

$$
f(x)=q(x) e^{-\left(a^{\prime} x^{2}+b^{\prime} x+c^{\prime}\right)}
$$

for some polynomial $q$.

In general we cannot say more about the form of $f$. If however, $f=q h_{0}$ with the standard Gaussian $h_{0}$, then we can reduce the problem of zero-free Wigner distributions to a problem of polynomials of two variables by means of the fundamental theorem of algebra for poly-analytic polynomials $[3,4]$.

Theorem 6.3 (Balk) Let $P(z, w)$ be a polynomial in two complex variables. If the exact degree of the poly-analytic polynomial $P(z, \bar{z})$ as a polynomial of two variables exceeds the exact degree of $P$ in one of these variables by a factor more than two, the $P$ has at least one zero.

In other words, let $n_{z}=\operatorname{deg}_{z} P, n_{\bar{z}}=\operatorname{deg}_{\bar{z}} P$, and $s=\operatorname{deg} P$. If $s>2 \min \left(n_{z}, n_{\bar{z}}\right)$, then $P$ has at least one zero. See $[3,4]$ for the proof.

From this we deduce the following consequence.

Proposition 6.4 Assume that $g=p h_{0}$ and $f=q h_{0}$ for some polynomials $p, q$. If $V_{g} f$ does not have any zeros, then $\operatorname{deg} p=\operatorname{deg} q$.

In other words, if $f$ and $g$ are finite linear combinations of Hermite functions, whose highest degrees differ, then the short-time Fourier transform $V_{g} f$ must have a zero. 
Proof Under the assumptions stated, $B f$ is a polynomial $Q$ and the poly-analytic Bargmann transform is then (for $N$ large enough)

$$
B_{g} f(z)=\sum_{k=0}^{N} \frac{1}{k !} Q^{(k)}(z) \overline{P^{(k)}(-\pi z)}
$$

is a poly-analytic polynomial. Here $\operatorname{deg}_{z} B_{g} f=\operatorname{deg} Q, \operatorname{deg}_{\bar{z}} B_{g} f=\operatorname{deg} P$, and $\operatorname{deg} B_{g} f=\operatorname{deg} P+\operatorname{deg} Q$. If $\operatorname{deg} P \neq \operatorname{deg} Q$, then $\operatorname{deg} B_{g} f>2 \min (\operatorname{deg} P, \operatorname{deg} Q)$, and by the fundamental theorem of algebra $B_{g} f$ must have a zero.

If the degree condition in the fundamental theorem is not satisfied, then a polyanalytic polynomial may not have any zeros, for instance, the polynomial $p(z, \bar{z})=$ $1+z \bar{z}$ does not have any zeros. Note, however, that the poly-analytic polynomials arising in Proposition 6.4 have a very special structure.

Let us consider the simple example of degree 1 polynomials. It is enough to consider $P(z)=-z+\pi b$ and $Q(z)=z+a$ with $a, b \in \mathbb{C}$. Then we are looking for a root of the polynomial

$$
Q(z) \overline{P(-\pi z)}+Q^{\prime}(z) \overline{P^{\prime}(-\pi z)}=\pi(z+a)(\bar{z}+\bar{b})-1 \text {. }
$$

Write $\zeta=\sqrt{\pi}(z+a)$ and $c=\sqrt{\pi}(\bar{b}-\bar{a})$, the equation becomes $\zeta(\bar{\zeta}+c)=1$. Writing $c$ in polar coordinates $c=\rho e^{i \theta}$ and a further change of variable $\zeta=\xi e^{-i \theta}$ yields the equation $\xi(\bar{\xi}+\rho)=1$ with $\rho \geq 0$. This equation has at least two real roots, namely $\frac{-\rho \pm \sqrt{\rho^{2}+4}}{2}$. This proves the following.

Proposition 6.5 If both $f$ and $g$ are linear combinations of $h_{0}, h_{1}$, then $W(f, g)$ has a zero, unless $f, g$ are both multiples of $h_{0}$.

In general, $B_{g} f$ may be written as a linear combination of various Laguerre polynomials or complex Hermite polynomials, see [10] or [16]. Thus $B_{g} f$ has a very special structure. One may therefore hope to prove the existence of a zero for the special poly-analytic polynomials of the form $B_{g} f$. Another simple example is constructed as follows: if $f=p h_{0}$ for some polynomial $p$ of degree at least 1 and $g=I f$ (i.e., $\left.g(x)=f(-x)=p(-x) h_{0}(x)\right)$, then $\left|V_{I f} f(2 z)\right|=W(f, f)$. The corresponding poly-analytic polynomial $B_{I f} f$ then has a zero by Hudson's theorem, although Balk's fundamental theorem of algebra does not apply.

\section{An example for bounded functions: the short-time Fourier transform with a rectangular window}

We next study a class of examples in which the decay properties are critical for the existence or non-existence of zeros of the short-time Fourier transform. For this purpose, we investigate the short-time Fourier transform of carefully constructed step functions. We consider the window $\chi=\mathbf{1}_{(0,1)}$ and step functions with discontinuities on the union $\mathbb{Z} \cup(\alpha+\mathbb{Z})$ for irrational $\alpha$. We will need a simple lemma first. 
Lemma 7.1 Let $0=a_{1}<a_{2}<\cdots<a_{n}<a_{n+1}=1$ and either $c_{1}>c_{2}>\cdots>$ $c_{n}>0$ or $0<c_{1}<c_{2}<\cdots<c_{n}$. Define $f$ on $(0,1)$ by

$$
f(x)=\sum_{k=1}^{n} c_{k} \mathbf{1}_{\left(a_{k}, a_{k+1}\right)}
$$

Then there exists $\xi \in \mathbb{R}$ such that

$$
\int_{0}^{1} f(x) e^{-2 \pi i x \xi} \mathrm{d} x=0
$$

if and only if $a_{2}, \ldots, a_{n} \in \mathbb{Q}$.

Proof It is enough to consider the case when $c_{j}$ is strictly decreasing. The case of increasing $c_{j}$ 's reduces to this case by changing $f(t)$ into $f(1-t)$. For $\xi=0$ we obtain

$$
\int_{0}^{1} f(x) e^{-2 \pi i x \xi} \mathrm{d} x=\int_{0}^{1} f(x) \mathrm{d} x=\sum_{k=1}^{n} c_{k}\left(a_{k+1}-a_{k}\right)>0 .
$$

For $\xi \neq 0$ we set $I(\xi)=2 \pi i \xi \int_{0}^{1} f(x) e^{2 \pi i x \xi} \mathrm{d} x=2 \pi i \xi \hat{f}(-\xi)$. Then

$$
\begin{aligned}
I(\xi) & =2 \pi i \xi \int_{0}^{1} f(x) e^{2 \pi i x \xi} \mathrm{d} x=\sum_{k=1}^{n} c_{k}\left(e^{2 \pi i a_{k+1} \xi}-e^{2 \pi i a_{k} \xi}\right) \\
& =-c_{1}+\sum_{k=2}^{n}\left(c_{k-1}-c_{k}\right) e^{2 \pi i a_{k} \xi}+c_{n} e^{2 \pi i \xi} \\
& =c_{1}\left(\sum_{k=2}^{n} \frac{c_{k-1}-c_{k}}{c_{1}} e^{2 \pi i a_{k} \xi}+\frac{c_{n}}{c_{1}} e^{2 \pi i \xi}-1\right) .
\end{aligned}
$$

Thus $\hat{f}(-\xi)=(2 \pi i \xi)^{-1} I(\xi)=0$, if and only if

$$
\sum_{k=2}^{n} \frac{c_{k-1}-c_{k}}{c_{1}} e^{2 \pi i a_{k} \xi}+\frac{c_{n}}{c_{1}} e^{2 \pi i \xi}=1
$$

Since $\left|e^{2 \pi i a_{k} \xi}\right|=1$ and

$$
\sum_{k=2}^{n} \frac{c_{k-1}-c_{k}}{c_{1}}+\frac{c_{n}}{c_{1}}=1
$$

with positive terms, (32) is a convex linear combination of points in the unit disc of $\mathbb{C}$. 
As 1 is an extreme point of the unit disc, (32) holds if and only if it is a convex combination of 1's. Therefore, $I(\xi)=0$ if and only if $e^{2 \pi i a_{k} \xi}=1, k=2, \ldots, n$, and $e^{2 \pi i \xi}=1$. This implies that $\xi \in \mathbb{Z} \backslash\{0\}$ and $a_{k} \xi \in \mathbb{Z} \backslash\{0\}, k=2, \ldots, n$. Thus, if one of the $a_{k}$ 's is irrational, then $\hat{f}(\xi) \neq 0$ for all $\xi \neq 0$. Conversely, if all $a_{k} \in \mathbb{Q}$, write $a_{k}=p_{k} / q_{k}, p_{k}, q_{k} \in \mathbb{Z}$ and take $\xi=q_{1} q_{2} \cdots q_{n}$ then $I(\xi)=0$. Note that this is true whatever the $c_{k}$ 's are.

The case $c_{k+1}=c_{k}$ does not need to be considered, as this amounts to remove $a_{k+1}$.

The proof of Lemma 7.1 no longer works when the sequence $c_{k}$ is not monotonic. Instead we prove the following.

Lemma 7.2 Let $\alpha \in(0,1) \backslash \mathbb{Q}$ and $0<b, c<d$. For each $a \in(0,1-\alpha)$ we define

$$
f_{a}(t)=b \mathbf{1}_{(0, a)}+d \mathbf{1}_{(a, a+\alpha)}+c \mathbf{1}_{(a+\alpha, 1)} .
$$

Then there exists $a \in(0,1-\alpha)$ and $\xi \in \mathbb{R}$, such that $\hat{f}_{a}(\xi)=0$.

Proof We may assume without loss of generality that $d=1$ and $0<b, c<1$.

For $\xi \neq 0$ we use again $I(\xi)=2 \pi i \xi \hat{f}(-\xi)$. The computation shows that

$$
\begin{aligned}
I(\xi) & =b\left(e^{2 \pi i a \xi}-1\right)+\left(e^{2 \pi i(a+\alpha) \xi}-e^{2 \pi i a \xi}\right)+c\left(e^{2 \pi i \xi}-e^{2 \pi i(a+\alpha) \xi}\right) \\
& =e^{2 \pi i a \xi}\left((1-c) e^{2 \pi i \alpha \xi}-(1-b)\right)+c e^{2 \pi i \xi}-b .
\end{aligned}
$$

We proceed in two steps. The first step consists in determining a set of $\xi$ 's independent of $a$ for which $I(\xi)$ may be 0 . Indeed, note that, if $I(\xi)=0$, then

$$
\left|(1-c) e^{-2 \pi i \alpha \xi}-(1-b)\right|^{2}=\left|c e^{-2 \pi i \xi}-b\right|^{2}
$$

or $(1-c)^{2}+(1-b)^{2}-2(1-c)(1-b) \cos 2 \pi \alpha \xi=c^{2}+b^{2}-2 b c \cos 2 \pi \xi$. We obtain that $I(\xi)=0$ implies the identity

$$
1-b-c=(1-b-c+b c) \cos 2 \pi \alpha \xi-b c \cos 2 \pi \xi
$$

Let $M=(1-b)(1-c)+b c=1-b-c+2 b c$. Clearly $1-b-c<M$ and $M+1-b-c=2(1-b)(1-c)>0$, thus $|1-b-c|<M$. Consider the function $\psi(\xi)=(1-b-c+b c) \cos 2 \pi \alpha \xi-b c \cos 2 \pi \xi$. Clearly, $|\psi(\xi)| \leq M$, and more importantly, since $\alpha \notin \mathbb{Q}, \psi$ is almost periodic and takes every value in $(-M, M)$ infinitely often. More precisely, for each $t \in(-M, M)$ there exists a uniformly discrete set $\Lambda_{t}$ such that, for every $\xi \in \Lambda_{t},(1-b-c+b c) \cos 2 \pi \alpha \xi-b c \cos 2 \pi \xi=t$ (see, e.g., [7]). Let $\Lambda:=\Lambda_{1-b-c}=\{\xi \in \mathbb{R}:(1-b-c+b c) \cos 2 \pi \alpha \xi-b c \cos 2 \pi \xi=1-b-c\}$.

Further, note that if, for some $\xi \in \Lambda$ we had $(1-c) e^{2 \pi i \alpha \xi}-(1-b)=b-c e^{2 \pi i \xi}=0$ then $b=|b|=\left|c e^{2 \pi i \xi}\right|=c$. Further $e^{2 \pi i \xi}=1$ and then $(1-c) e^{2 \pi i \alpha \xi}-(1-b)=0$ reduces to $e^{2 \pi i \alpha \xi}=1$ which is impossible as $\alpha \notin \mathbb{Q}$. 
Therefore, for $\xi \in \Lambda$ we must have $\left|(1-c) e^{2 \pi i \alpha \xi}-(1-b)\right|=\left|b-c e^{2 \pi i \xi}\right| \neq 0$. Thus there exists $\theta_{\xi} \in(0,2 \pi]$ such that

$$
\frac{b-c e^{2 \pi i \xi}}{(1-c) e^{2 \pi i \alpha \xi}-(1-b)}=e^{i \theta_{\xi}} .
$$

In the second step we find an appropriate value for $a$ as follows: As $\Lambda$ is discrete and infinite, $\Lambda$ contains arbitrarily large $\xi$ 's, and there exists $\xi$ such that $a:=\theta_{\xi} / \xi \in$ $(0,1-\alpha)$ and then

$$
\frac{b-c e^{2 \pi i \xi}}{(1-c) e^{2 \pi i \alpha \xi}-(1-b)}=e^{i \theta_{\xi}}=e^{2 \pi i a \xi} .
$$

It follows that $I(\xi)=0$ thus $\hat{f}_{a}(\xi)=0$.

The following result shows that the construction of zero-free Wigner distributions with $\chi=\mathbf{1}_{(0,1)}$ might be rather delicate. We assume that $f$ is a step function with jumps on $\mathbb{Z} \cup(\alpha+\mathbb{Z})$ for $\alpha \notin \mathbb{Q}$, then every short-time Fourier transform $V_{g} f$ with $f \in L^{2}(\mathbb{R})$ possesses a zero. However, if we "slightly" enlarge the function space and also consider bounded step functions, then we can produce a zero-free short-time Fourier transform $V_{g} f$.

Theorem 7.3 Let $\left(c_{k}\right)_{k \in \mathbb{Z}} \subset \mathbb{R}$ be a bounded positive sequence. Let $\alpha \in(0,1) \backslash \mathbb{Q}$ and define the sequence $\left(a_{k}\right)$ by $a_{2 k}=k, a_{2 k+1}=k+\alpha$. Let $\chi=\mathbf{1}_{(0,1)}$ and

$$
\varphi=\sum_{k \in \mathbb{Z}} c_{k} \mathbf{1}_{\left(a_{k}, a_{k+1}\right)}
$$

(i) If $\left(c_{k}\right)_{k \in \mathbb{Z}}$ is monotonic, then $\varphi \in L^{\infty}(\mathbb{R})$ and $V_{\chi} f$ never vanishes.

(ii) If $\varphi \in L^{p}(\mathbb{R})$ for $1 \leq p<\infty$, then there exists $(x, \xi) \in \mathbb{R}^{2}$ such that $V_{\chi} f(x, \xi)=0$.

Proof Clearly, for $1 \leq p \leq \infty, \varphi \in L^{p}(\mathbb{R})$, if and only if $\left(c_{k}\right) \in \ell^{p}(\mathbb{Z})$. We write

$$
\varphi=\sum_{k \in \mathbb{Z}}\left(c_{2 k} \mathbf{1}_{(k, k+\alpha)}+c_{2 k+1} \mathbf{1}_{(k+\alpha, k+1)}\right) .
$$

Then the function $\varphi(t) \chi(t-x)$ is a step function consisting of three terms. To describe the correct terms, we write $x=j+u$ with $j \in \mathbb{Z}$ and $u \in[0,1)$ and distinguish two cases.

Case 1. If $u \in[0, \alpha)$, then

$$
\begin{aligned}
& V_{\chi} \varphi(x, \xi) \\
& \quad=\int_{\mathbb{R}}\left(c_{2 j} \mathbf{1}_{(j+u, j+\alpha)}(t)+c_{2 j+1} \mathbf{1}_{(j+\alpha, j+1)}(t)+c_{2 j+2} \mathbf{1}_{(j+1, j+1+u)}(t)\right) e^{-2 \pi i t \xi} \mathrm{d} t
\end{aligned}
$$




$$
\begin{aligned}
= & e^{-2 \pi i(j+u) \xi} \int_{0}^{1}\left(c_{2 j} \mathbf{1}_{(0, \alpha-u)}(t)+c_{2 j+1} \mathbf{1}_{(\alpha-u, 1-u)}(t)\right. \\
& \left.+c_{2 j+2} \mathbf{1}_{(1-u, 1)}(t)\right) e^{-2 \pi i t \xi} \mathrm{d} t .
\end{aligned}
$$

We observe right away that at least one of the endpoints $\alpha-u$ or $1-u$ must be irrational, since $\alpha \notin \mathbb{Q}$.

Case 2. If $u \in[\alpha, 1)$, then

$$
\begin{aligned}
& V_{\chi} \varphi(x, \xi) \\
& =\int_{\mathbb{R}}\left(c_{2 j+1} \mathbf{1}_{(j+u, j+1)}(t)+c_{2 j+2} \mathbf{1}_{(j+1, j+1+\alpha)}(t)+c_{2 j+2} \mathbf{1}_{(j+1+\alpha, j+1+u)}(t)\right) e^{-2 \pi i \xi t} \mathrm{~d} t \\
& =e^{-2 \pi i \xi(j+u)} \int_{0}^{1}\left(c_{2 j+1} \mathbf{1}_{(0,1-u)}(t)+c_{2 j+2} \mathbf{1}_{(1-u, 1-u+\alpha)}(t)+c_{2 j+3} \mathbf{1}_{(1-u+\alpha, 1)}(t)\right) e^{-2 \pi i t \xi} \mathrm{d} t .
\end{aligned}
$$

Again, at least one of the endpoints $1-u$ and $1-u+\alpha$ must be irrational, because $\alpha \notin \mathbb{Q}$.

Proof of (i): If the coefficient sequence $\left(c_{j}\right)$ is monotonic, then Lemma 7.1 is applicable, and we conclude that $V_{\chi} \varphi(x, \xi) \neq 0$ for all $\xi \in \mathbb{R}$ and all $x \in \mathbb{R}$.

Proof of (ii): Assume now that $\varphi \in L^{p}(\mathbb{R})$ for $p<\infty$, then $\left(c_{j}\right) \in \ell^{p}$ cannot be monotonic. Thus there exists $j_{0}$ such that either $c_{2 j_{0}}, c_{2 j_{0}+2}<c_{2 j_{0}+1}$ or $c_{2 j_{0}+1}, c_{2 j_{0}+3}<c_{2 j_{0}+2}$. In the former case we choose $x=j_{0}+u$ for $u \in(0, \alpha)$ (to be determined in a moment) and we work with (36). In the latter case we choose $x=j_{0}+u$ for $u \in(\alpha, 1)$ and work with (37). In both cases the length of the middle interval $(\alpha-u, 1-u)$ and $(1-u, 1-u+\alpha)$ is irrational. According to Lemma 7.2, there exists suitable $u$ and $\xi \in \mathbb{R}$ such that $V_{\chi} \varphi\left(j_{0}+u, \xi\right)=0$.

Acknowledgements Open access funding provided by Austrian Science Fund (FWF). The authors would like to thank the Erwin Schrödinger Institute for its hospitality and great conditions during the work on this paper. Special thanks go to Martin Ehler for his help with Mathematica and his helpful discussions and to Andrii Bondarenko who showed us Example 5. K. G. was partially supported by the Grant P31887-N32 of the Austrian Science Fund FWF. P. J. acknowledges that this study has been carried out with financial support from the French State, managed by the French National Research Agency (ANR) in the frame of the "Investments for the future" Programme IdEx Bordeaux - CPU (ANR-10-IDEX-03-02). E. M. was supported by Norwegian Research Council under Project 275113 and by the National Science Foundation under Grant No. DMS-1638352.

Open Access This article is distributed under the terms of the Creative Commons Attribution 4.0 International License (http://creativecommons.org/licenses/by/4.0/), which permits unrestricted use, distribution, and reproduction in any medium, provided you give appropriate credit to the original author(s) and the source, provide a link to the Creative Commons license, and indicate if changes were made.

\section{References}

1. Abreu, L.D.: Sampling and interpolation in Bargmann-Fock spaces of polyanalytic functions. Appl. Comput. Harmon. Anal. 29(3), 287-302 (2010)

2. Balk, M.B.: Entire poly-analytic functions with a bounded set of zeros. Izv. Akad. Nauk Armjan. SSR Ser. Mat. 1(5), 341-357 (1966) 
3. Balk, M.B.: The fundamental theorem of algebra for polyanalytic polynomials. Litovsk. Mat. Sb. 8, 401-404 (1968)

4. Balk, M.B.: Polyanalytic Functions. Mathematical Research, vol. 63. Akademie-Verlag, Berlin (1991)

5. Bayer, D.: Bilinear time-frequency distributions and pseudo differential operators. Ph.D. thesis, University of Vienna (2010)

6. Bayer, D., Gröchenig, K.: Time-frequency localization operators and a Berezin transform. Integr. Equ. Oper. Theory 82(1), 95-117 (2015)

7. Besicovitch, A.S.: Almost Periodic Functions. Cambridge University Press, Cambridge (1932)

8. Brekke, S., Seip, K.: Density theorems for sampling and interpolation in the Bargmann-Fock space. III. Math. Scand. 73(1), 112-126 (1993)

9. de Gosson, M.A.: Symplectic Methods in Harmonic Analysis and in Mathematical Physics. PseudoDifferential Operators, vol. 7. Theory and Applications. Springer Basel AG, Basel (2011)

10. Folland, G.B.: Harmonic Analysis in Phase Space. Princeton University Press, Princeton (1989)

11. Gantmacher, F.R.: The Theory of Matrices, vol. II. Chelsea Publishing, New York (1959)

12. Gröchenig, K.: Foundations of Time-Frequency Analysis. Birkhäuser Boston Inc., Boston (2001)

13. Gröchenig, K., Lyubarskii, Y.: Gabor (super)frames with Hermite functions. Math. Ann. 345(2), 267286 (2009)

14. Gröchenig, K., Zimmermann, G.: Hardy's theorem and the short-time Fourier transform of Schwartz functions. J. Lond. Math. Soc. 63, 205-214 (2001)

15. Hudson, R.L.: When is the Wigner quasi-probability density non-negative? Rep. Math. Phys. 6(2), 249-252 (1974)

16. Ismail, M.E.H.: Analytic properties of complex Hermite polynomials. Trans. Am. Math. Soc. 368(2), 1189-1210 (2016)

17. Janssen, A.J.E.M.: A note on Hudson's theorem about functions with nonnegative Wigner distributions. SIAM J. Math. Anal. 15(1), 170-176 (1984)

18. Janssen, A.J.E.M.: Bilinear phase-plane distribution functions and positivity. J. Math. Phys. 26(8), 1986-1994 (1985)

19. Janssen, A.J.E.M.: Some Weyl-Heisenberg frame bound calculations. Indag. Math. 7, 165-182 (1996)

20. Katkova, O.M., Vishnyakova, A.M.: A sufficient condition for a polynomial to be stable. J. Math. Anal. Appl. 347(1), 81-89 (2008)

21. Khavinson, D., Neumann, G.: From the fundamental theorem of algebra to astrophysics: a "harmonious" path. Notices Am. Math. Soc. 55(6), 666-675 (2008)

22. Kiukas, J., Lahti, P., Schultz, J., Werner, R.F.: Characterization of informational completeness for covariant phase space observables. J. Math. Phys. 53(10), 102103 (2012)

23. Lieb, E.H.: Integral bounds for radar ambiguity functions and Wigner distributions. J. Math. Phys. 31(3), 594-599 (1990)

24. Luef, F., Skrettingland, E.: Convolutions for Berezin quantization and Berezin-Lieb inequalities. J. Math. Phys. 59(2), 023502 (2018)

25. Macdonald Function. Encyclopedia of Mathematics. http://www.encyclopediaofmath.org

26. MacDonald, H.M.: Zeroes of the Bessel functions. Proc. Lond. Math. Soc. 30, 165-179 (1898/99)

27. Ostrovs' kiř, I.V.: A theorem of M. B. Balk. Mat. Fiz. i Funkcional. Anal. Vyp. 1, 191-202 (1969)

28. Schoenberg, I.J.: On Pólya frequency functions. I. The totally positive functions and their Laplace transforms. J. Analyse Math. 1, 331-374 (1951)

29. Strelitz, S.: On the Routh-Hurwitz problem. Am. Math. Monthly 84, 542-544 (1977)

30. Vasilevski, N.L.: Poly-Fock Spaces. In Differential Operators and Related Topics, Vol. I (Odessa, 1997), Volume 117 of Operator Theory: Advances and Applications, pp. 371-386. Birkhäuser, Basel (2000)

31. Watson, G.N.: A Treatise on the Theory of Bessel Functions. Cambridge Mathematical Library. Cambridge University Press, Cambridge (1995). [Reprint of the second (1944) edition]

Publisher's Note Springer Nature remains neutral with regard to jurisdictional claims in published maps and institutional affiliations. 